\title{
Optimal Cost Allocation in Centralized and Decentralized Detection Problems
}

\author{
Eray Laz ${ }^{*, \natural}$ and Sinan Gezici* \\ * Department of Electrical and Electronics Engineering, Bilkent University, Ankara, 06800, Turkey \\ $\natural$ Radar, Electronic Warfare and Intelligence Division, ASELSAN Inc., Ankara, Turkey \\ eray@ee.bilkent.edu.tr, gezici@ee.bilkent.edu.tr
}

\begin{abstract}
The optimal cost allocation problem is proposed for centralized and decentralized detection systems in the presence of cost constrained measurements, where the aim is to minimize the probability of error of a given detection system under a total cost constraint. The probability of error expressions are obtained for centralized and decentralized detection systems, and the optimal cost allocation strategies are provided. In addition, special cases are investigated in the presence of Gaussian observations and measurement noise. The solutions of the proposed problems specify the optimal allocation of the cost budget among various measurement devices (sensors) to achieve the optimum detection performance. Numerical examples are presented to discuss the implications of the results.
\end{abstract}

Index Terms-Bayes risk, hypothesis testing, measurement cost, (de)centralized detection, sensor network.

\section{INTRODUCTION}

In this manuscript, centralized and decentralized detection systems are studied in the presence of cost constrained measurements. In such systems, decisions are performed based on measurements collected by multiple sensors, the qualities of which are determined by assigned cost values. The aim is to develop optimal cost allocation strategies in a Bayesian framework under a total cost constraint. In the case of centralized detection, a set of geographically separated sensors transmit their complete measurements to a fusion center, and the fusion center selects one of the hypotheses [1]. On the other hand, in decentralized detection, sensors send a summary of their measurements to the fusion center [2]. For quantifying the costs of measurement devices (sensors), the model in [3] is employed in this study. According to [3], the cost of a measurement device is basically determined by the number of amplitude levels that it can reliably distinguish.

Detection and estimation problems considering system resource constraints have been studied in the literature [4]-[10]. In [4], measurement cost minimization is studied under various estimation accuracy constraints. In [5], optimal distributed detection strategies for wireless sensor networks are studied by considering network resource constraints, where it is assumed that observations at the sensors are spatially and temporally independent and identically distributed (i.i.d.). Two types of constraints are taken into consideration related to the transmission power and the communication channel. For the communication channel, there exist two options, which are multiple access and parallel access channels. It is proved that using a multiple access channel with analog communication of local likelihood ratios (soft decisions) is asymptotically optimal when each sensor communicates with a constant power [5]. In [6], binary decentralized detection problem is investigated under the constraint of wireless channel capacity. It is shown that having a set of identical sensors is asymptotically optimal when the observations conditioned on the hypothesis are i.i.d. and the number of observations per sensors goes infinity. In [7], a decentralized detection problem is studied, where the sensors have side information that affects the statistics of their measurements and the network has a cost constraint. The study examines wireless sensor networks with a cost constraint and a capacity constraint separately. In both cases, the error exponent is minimized under the specified constraints. [7] produces a similar result to that in [6] for the capacity constraint case. In addition, [7] and [8] have the same results for the power constraint case. It is found that having identical sensors which use the same transmission scheme is asymptotically optimal when the observations are conditionally independent given the state of the nature. In [9], decentralized detection is studied with constraints on the expected cost due to transmissions from sensor nodes and the measurement at each local sensor, where it is shown that optimum detection performance can be obtained via randomization.

Based on the cost function proposed in [3] for obtaining measurements, various studies have been performed on estimation with cost constraints [4], [10]. In particular, [4] considers the costs of measurements and aims to minimize the total cost under various estimation accuracy constraints. In [10], average Fisher information maximization is studied under cost constrained measurements. On the other hand, [11] investigates the tradeoff between reducing the measurement cost and keeping the estimation accuracy within acceptable levels in continuous time linear filtering problems. In [12], the channel switching problem is studied, where the aim is to minimize the probability of error between a transmitter and a receiver that are connected via multiple channels and only one channel can be used at a given time. In that study, a logarithmic cost function similar to that in [3] is employed for specifying the cost of using a certain channel.

Although costs of measurements have been considered in various estimation and channel switching problems such as [4], [10]-[12], there exist no studies in the literature that consider the optimization of centralized and decentralized detection systems in the presence of cost constrained measurements based on a specific cost function as in [3]. In this study, we first consider the centralized detection problem and propose a general formulation for allocating the cost budget to measurement devices in order to achieve the optimum performance in the Bayesian framework. Also, a closed-form expression is obtained for binary hypothesis testing with Gaussian observa- 
tions. Then, we investigate the decentralized detection problem with some common fusion rules, and present a generic formulation that aims to minimize the probability of error by optimally allocating the cost budget to measurement devices. A numerical solution is proposed for binary hypothesis testing with Gaussian observations. As convexity is an important property for the optimization problems, the convexity property is explored for the case of two measurement devices. Finally, numerical examples are presented to demonstrate the results.

\section{Cost Allocation for Centralized Detection}

In centralized detection problems, all sensor nodes transmit their observations to the fusion center, and the decision is made in the fusion center based on the data from all the sensors. The system model for the centralized detection is illustrated in Fig. 1.

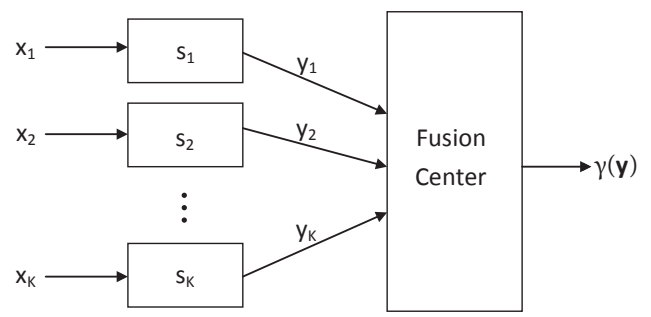

Fig. 1. Centralized detection system model.

As shown in Fig. $1, x_{1}, x_{2}, \ldots, x_{K}$ represent the scalar observations, and $\mathrm{s}_{1}, \mathrm{~s}_{2}, \ldots, \mathrm{s}_{K}$ denote the sensors by which the measurements are taken. The measurement at sensor $i$ is represented as $y_{i}=x_{i}+m_{i}$, where $m_{i}$ is the measurement noise. The measurement $\mathbf{y} \in \mathbb{R}^{K}$ is processed by the fusion center to produce the final decision $\gamma(\mathbf{y})$, where $\mathbf{y}=\left[y_{1}, y_{2}, \ldots, y_{K}\right]^{T}$ and $\gamma(\mathbf{y})$ takes values from $\{0,1, \ldots, M-1\}$ for $M$-ary hypothesis testing.

In the Bayesian hypothesis-testing framework, the optimum decision rule is the one that minimizes the Bayes risk, which is defined as the average of the conditional risks [13]. The conditional risk for a decision rule $\delta(\cdot)$ when the state of nature is $H_{j}$ is given by

$$
R_{j}(\delta)=\sum_{i=0}^{M-1} \tilde{c}_{i j} P_{j}\left(\Gamma_{i}\right)
$$

where $\tilde{c}_{i j}$ is the cost of choosing hypothesis $H_{i}$ when the state of nature is $H_{j}$, and $P_{j}\left(\Gamma_{i}\right)$ is the probability of deciding hypothesis $H_{i}$ when $H_{j}$ is correct, with $\Gamma_{i}$ denoting the decision region for hypothesis $H_{i}$. Then, the Bayes risk can be expressed as

$$
r(\delta)=\sum_{j=0}^{M-1} \pi_{j} R_{j}(\delta)
$$

where $\pi_{j}$ is the prior probability of hypothesis $H_{j}$. For the values of $\tilde{c}_{i j}$, uniform cost assignment (UCA) is commonly employed, which is stated as [13]

$$
\tilde{c}_{i j}=\left\{\begin{array}{ll}
0, & \text { if } i=j \\
1, & \text { if } i \neq j
\end{array} .\right.
$$

For UCA, the Bayes rule, which minimizes the Bayes risk specified by (1) and (2), reduces to choosing the hypothesis with the maximum a-posteriori probability (MAP), and the corresponding Bayes risk can be stated, after some manipulation, as

$$
r\left(\delta_{B}\right)=1-\int_{\mathbb{R}^{K}} \max _{l=\{0,1, \ldots, M-1\}} \pi_{l} p_{l}(\mathbf{y}) d \mathbf{y}
$$

where $\delta_{B}$ denotes the Bayes rule, and $p_{l}(\mathbf{y})$ is the probability distribution of $\mathbf{y}$ under hypothesis $H_{l}$ [13].

In this section, the aim is to perform the optimal cost allocation among the sensors in Fig. 1 in order to minimize the Bayes risk expression in (4) under a total cost constraint. The cost of measuring the $i$ th component of the observation vector, $x_{i}$, is given by $C_{i}=0.5 \log _{2}\left(1+\sigma_{x_{i}}^{2} / \sigma_{m_{i}}^{2}\right)$, where $\sigma_{x_{i}}^{2}$ is the variance of $x_{i}$ and $\sigma_{m_{i}}^{2}$ is the variance of the noise introduced by the $i$ th sensor [3]. Then, the total cost is expressed as

$$
C=\sum_{i=1}^{K} C_{i}=\frac{1}{2} \sum_{i=1}^{K} \log _{2}\left(1+\frac{\sigma_{x_{i}}^{2}}{\sigma_{m_{i}}^{2}}\right) .
$$

The cost function for each sensor is monotonically decreasing, nonnegative, and convex with respect to $\sigma_{m_{i}}^{2}$ for $\forall \sigma_{m_{i}}^{2}>0$ and $\forall \sigma_{x_{i}}^{2}>0$. (The convexity property of the cost function can easily be shown by examining the Hessian matrix [14].) In addition, when the measurement noise variance is low, the cost is high since the number of amplitude levels that the device can distinguish gets high [3]. When $\sigma_{m_{i}}^{2}$ goes to infinity, the cost converges to zero and when $\sigma_{m_{i}}^{2}$ goes to zero, the cost approaches infinity.

Based on (4) and (5), the following optimization problem is proposed for centralized detection problems:

$$
\begin{array}{ll}
\underset{\left\{\sigma_{m_{i}}^{2}\right\}_{i=1}^{K}}{\max } & \int_{\mathbb{R}^{K}} \underset{l=\{0,1, \ldots, M-1\}}{\max } \pi_{l} p_{l}(\mathbf{y}) d \mathbf{y} \\
\text { subject to } & \frac{1}{2} \sum_{i=1}^{K} \log _{2}\left(1+\frac{\sigma_{x_{i}}^{2}}{\sigma_{m_{i}}^{2}}\right) \leq C_{T}
\end{array}
$$

where $C_{T}$ is the (total) cost constraint. Hence, the optimal allocation of the measurement noise variances, $\sigma_{m_{i}}^{2}$, (equivalently, the costs, $C_{i}$ ) is to be performed under the total cost constraint. It is also noted that the maximization of the objective function in (6) corresponds to the minimization of the Bayes risk in (4), which represents the probability of error for the Bayes rule. When the optimization problem proposed in (6) is solved, the optimum cost values for the measurement devices (sensors) are obtained and these values achieve the optimum performance for centralized detection.

In practical systems, the observations, $\mathbf{x}=\left[x_{1}, \ldots, x_{K}\right]^{T}$, are independent of the measurement noise, $\mathbf{m}=$ $\left[m_{1}, \ldots, m_{K}\right]^{T}$. Hence, the conditional probability density function (PDF) of the measurement vector when hypothesis $H_{l}$ is true can be obtained as the convolution of the PDFs of $\mathbf{m}$ and $\mathbf{x}$ as follows:

$$
p_{l}(\mathbf{y})=\int_{\mathbb{R}^{K}} p_{\mathbf{M}}(\mathbf{m}) p_{\mathbf{X}}\left(\mathbf{y}-\mathbf{m} \mid H_{l}\right) d \mathbf{m} .
$$

In addition, if the sensors have independent noise, $p_{\mathbf{M}}(\mathbf{m})$ can be expressed as $p_{\mathbf{M}}(\mathbf{m})=p_{M_{1}}\left(m_{1}\right) \cdots p_{M_{K}}\left(m_{K}\right)$.

As a special case, a centralized binary hypothesis-testing problem is investigated in the presence of Gaussian observations and measurement noise, which is a common scenario 
in practice. In this case, the distribution of observation $\mathbf{x}$ under hypothesis $H_{0}$ is Gaussian with mean vector $\boldsymbol{\mu}_{\mathbf{0}}$ and covariance matrix $\boldsymbol{\Sigma}$, which is denoted by $\mathcal{N}\left(\boldsymbol{\mu}_{\mathbf{0}}, \boldsymbol{\Sigma}\right)$. Similarly, $\mathbf{x}$ is distributed as $\mathcal{N}\left(\boldsymbol{\mu}_{\mathbf{1}}, \boldsymbol{\Sigma}\right)$ under hypothesis $H_{1}$. In addition, the measurement noise vector, $\mathbf{m}$, is distributed as $\mathcal{N}\left(\mathbf{0}, \boldsymbol{\Sigma}_{\boldsymbol{m}}\right)$, where $\boldsymbol{\Sigma}_{\boldsymbol{m}}=\operatorname{diag}\left\{\sigma_{m_{1}}^{2}, \sigma_{m_{2}}^{2}, \ldots, \sigma_{m_{K}}^{2}\right\}$; that is, the measurement noise is independent for different sensors [3]. Considering that $\mathbf{x}$ and $\mathbf{m}$ are independent, the distribution of the measurement, $\mathbf{y}=\mathbf{x}+\mathbf{m}$, is denoted by $\mathcal{N}\left(\boldsymbol{\mu}_{\mathbf{0}}, \boldsymbol{\Sigma}+\boldsymbol{\Sigma}_{\boldsymbol{m}}\right)$ under hypothesis $H_{0}$ and by $\mathcal{N}\left(\boldsymbol{\mu}_{\mathbf{1}}, \boldsymbol{\Sigma}+\boldsymbol{\Sigma}_{\boldsymbol{m}}\right)$ under $H_{1}$.

For the hypothesis-testing problem specified in the previous paragraph, the Bayes risk corresponding to the Bayes rule can be obtained as follows in the case of UCA [13, Chapter 3]:

$$
r\left(\delta_{B}\right)=\pi_{0} Q\left(\frac{\ln \left(\pi_{0} / \pi_{1}\right)}{d}+\frac{d}{2}\right)+\pi_{1} Q\left(\frac{d}{2}-\frac{\ln \left(\pi_{0} / \pi_{1}\right)}{d}\right)
$$

where $d \triangleq \sqrt{\left(\boldsymbol{\mu}_{\mathbf{1}}-\boldsymbol{\mu}_{\mathbf{0}}\right)^{T}\left(\boldsymbol{\Sigma}+\boldsymbol{\Sigma}_{\boldsymbol{m}}\right)^{-1}\left(\boldsymbol{\mu}_{\mathbf{1}}-\boldsymbol{\mu}_{\mathbf{0}}\right)}$ and $Q(x)=(1 / \sqrt{2 \pi}) \int_{x}^{\infty} \mathrm{e}^{-0.5 t^{2}} d t$ denotes the $Q$-function. It can be shown that the derivative of $r\left(\delta_{B}\right)$ in (8) with respect to $d$ is negative for all values of $d$; hence, $r\left(\delta_{B}\right)$ is a monotone decreasing function of $d$. Therefore, the minimization of $r\left(\delta_{B}\right)$ can be achieved by maximizing $d$. If the observations are assumed to be independent; that is, if $\boldsymbol{\Sigma}=\operatorname{diag}\left\{\sigma_{x_{1}}^{2}, \sigma_{x_{2}}^{2}, \ldots, \sigma_{x_{K}}^{2}\right\}$, then $d$ can be expressed as $d=\sqrt{\sum_{i=1}^{K} \frac{\mu_{i}^{2}}{\sigma_{x_{i}}^{2}+\sigma_{m_{i}}^{2}}}$, where $\mu_{i}$ represents the $i$ th component of the vector $\boldsymbol{\mu}_{1}-\boldsymbol{\mu}_{0}$. Hence, the optimization problem in (6) for this case is stated as follows:

$$
\begin{array}{ll}
\max _{\left\{\sigma_{m_{i}}^{2}\right\}_{i=1}^{K}} & \sum_{i=1}^{K} \frac{\mu_{i}^{2}}{\sigma_{x_{i}}^{2}+\sigma_{m_{i}}^{2}} \\
\text { subject to } & \frac{1}{2} \sum_{i=1}^{K} \log _{2}\left(1+\frac{\sigma_{x_{i}}^{2}}{\sigma_{m_{i}}^{2}}\right) \leq C_{T}
\end{array}
$$

The objective function in (9) is convex with respect to $\sigma_{m_{i}}^{2}$ for $\forall \sigma_{m_{i}}^{2}>0$ and $\forall \sigma_{x_{i}}^{2}>0$ since the Hessian matrix of the objective function, $\mathbf{H}=\operatorname{diag}\left\{2 \mu_{1}^{2} /\left(\sigma_{x_{1}}^{2}+\sigma_{m_{1}}^{2}\right)^{3}, 2 \mu_{2}^{2} /\left(\sigma_{x_{2}}^{2}+\right.\right.$ $\left.\left.\sigma_{m_{2}}^{2}\right)^{3}, \ldots, 2 \mu_{K}^{2} /\left(\sigma_{x_{K}}^{2}+\sigma_{m_{K}}^{2}\right)^{3}\right\}$, is positive definite. Since a convex objective function is maximized over a convex set, the solution lies at the boundary [10], [15]. Therefore, the constraint function becomes an equality constraint and the optimization problem can be solved by using the Lagrange multipliers method [14], [15]. Based on this approach, the optimal cost allocation algorithm is obtained as follows:

$$
\sigma_{m_{i}}^{2}= \begin{cases}\frac{\sigma_{x_{i}}^{4}}{\mu_{i}^{2} \alpha-\sigma_{x_{i}}^{2}} & \text { if } \sigma_{x_{i}}^{2}<\mu_{i}^{2} \alpha \\ \infty & \text { if } \sigma_{x_{i}}^{2} \geq \mu_{i}^{2} \alpha\end{cases}
$$

with

$$
\alpha=\left(2^{2 C_{T}} \prod_{i \in S_{K}} \frac{\sigma_{x_{i}}^{2}}{\mu_{i}^{2}}\right)^{1 /\left|S_{K}\right|}
$$

where set $S_{K}$ is given by $S_{K}=\left\{i \in\{1,2, \ldots, K\}: \sigma_{m_{i}}^{2} \neq\right.$ $\infty\}$, and $\left|S_{K}\right|$ represents the number of elements in the set $S_{K}$. The algorithm in (10) implies that if the observation variance $\sigma_{x_{i}}^{2}$ is greater than $\mu_{i}^{2} \alpha$, the variance of the measurement

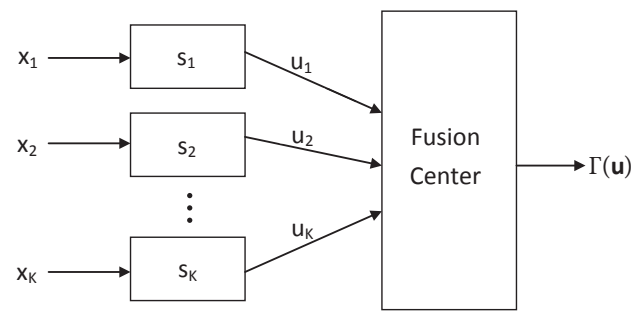

Fig. 2. Decentralized detection system model.

device (sensor) is set to infinity; that is, the observation is not measured at all, and the cost of the measurement device is zero. If the observation variance is smaller than the specified threshold, the variance of the measurement noise is calculated according to the expression in (10), which states that if the observation variance is low, the variance of the measurement device is assigned to be low. In other words, if the observation variance is low, a device with a high cost is considered to take measurements. Moreover, if the difference between the means of the observations for the two hypotheses, $\mu_{i}$, is high and $\sigma_{x_{i}}^{2}<\mu_{i}^{2} \alpha$ is satisfied, a low measurement noise variance is assigned to the measurement device. If $\mu_{i}$ is close to zero such that $\sigma_{x_{i}}^{2} \geq \mu_{i}^{2} \alpha$, a measurement device with zero cost is considered. Apart from this, if the observations are i.i.d. given the hypothesis, the variances of the measurement devices are chosen as equal, meaning that all the devices are required to have equal costs in order to achieve the optimum performance. The variances of the measurement devices become $\sigma_{m}^{2}=\sigma_{x}^{2} /\left(2^{2 C_{T} / K}-1\right)$ for i.i.d. observations.

\section{Cost Allocation for Decentralized Detection}

In contrast to centralized detection, local sensors send a summary of their observations to the fusion center in decentralized detection. For binary hypothesis-testing, local sensors can send their binary decisions about the true hypothesis ( 0 or 1) to the fusion center. The fusion center collects the binary decisions of the sensors and decides on the hypothesis. The fusion center can employ, e.g., OR, AND, or majority rules [16] as discussed in the following. The system model in this scenario is presented in Fig. 2. As in centralized detection, sensor $i, \mathrm{~s}_{i}$, measures the observation as $y_{i}=x_{i}+m_{i}$. Then, the sensors make local decisions about one of the two hypotheses as $\gamma_{i}\left(y_{i}\right)=u_{i}$, where $u_{i}$ is equal to 0 for hypothesis $H_{0}$ and 1 for hypothesis $H_{1}$. The outputs of the sensors, $u_{1}, u_{2}, \ldots, u_{K}$, are provided as inputs to the fusion center, which makes the final decision denoted by $\Gamma(\mathbf{u})$. The fusion rule that is employed in this section is the majority rule [16]. The majority rule is optimal when the noise components of the sensors are i.i.d., the hypotheses are equally likely, and the observations are i.i.d. and independent of the noise of the sensors [17]. The expression for the majority rule is given by

$$
\Gamma\left(u_{1}, u_{2}, \ldots, u_{K}\right)= \begin{cases}1, & \text { if } \sum_{i=1}^{K} u_{i} \geq t \\ 0, & \text { if } \sum_{i=1}^{K} u_{i}<t\end{cases}
$$

with $t=\lfloor K / 2\rfloor+1$, where $\lfloor\cdot\rfloor$ represents the floor operator that maps a real number to the largest integer lower than or equal to itself. Altough the majority rule is considered in 
the following analysis, the results can easily be extended for generic integer values of $t$ in (12). (For $t=1$ and $t=K$, the rule in (12) reduces to the OR fusion rule and the AND fusion rule, respectively.)

Considering independent but not necessarily identically distributed measurements ( $y_{i}$ 's), the probability of error (i.e., the Bayes risk for UCA) for the fusion rule in (12) can be calculated as

$$
r(\Gamma)=\pi_{0} \sum_{z=t}^{K} \sum_{c=1}^{\left(\begin{array}{c}
K \\
z
\end{array}\right)} \prod_{i=1}^{K} p_{l_{(z, c, i)}^{i} 0}+\pi_{1} \sum_{z=0}^{t-1} \sum_{c=1}^{\left(\begin{array}{c}
K \\
z
\end{array}\right)} \prod_{i=1}^{K} p_{l_{(z, c, i)}^{i}}
$$

where $p_{l_{(z, c, i)} j}^{i}$ denotes, for the $i$ th sensor, the probability of choosing hypothesis $H_{l_{(z, c, i)}}$ when hypothesis $H_{j}$ is true, and $l_{(z, c, i)}$ corresponds to the element at the $c$ th row and the $i$ th column of matrix $\mathbf{L}(z)$, which has a dimension of $\left(\begin{array}{c}K \\ z\end{array}\right) \times K$ and is formed as follows: The numbers of 1's and 0's in a row are $z$ and $K-z$, respectively, and the rows of the matrix contain all possible combinations of $z 1$ 's and $K-z 0$ 's. (Although matrix $\mathbf{L}(z)$ is not unique (e.g., the orders of the rows can be changed), all the $\mathbf{L}(z)$ matrices result in the same probability of error in (13).)

In the decentralized detection framework, the aim is to minimize the probability of error in (13) under the total cost constraint; that is,

$$
\begin{aligned}
& \min _{\left\{\sigma_{m_{i}}^{2}\right\}_{i=1}^{K}} \pi_{0} \sum_{z=t}^{K} \sum_{c=1}^{K} \prod_{i=1}^{K} p_{l_{(z, c, i} 0}^{i}+\pi_{1} \sum_{z=0}^{t-1} \sum_{c=1}^{K} \prod_{i=1}^{K} p_{l_{(z, c, i}}^{i} 1 \\
& \text { subject to } \frac{1}{2} \sum_{i=1}^{K} \log _{2}\left(1+\frac{\sigma_{x_{i}}^{2}}{\sigma_{m_{i}}^{2}}\right) \leq C_{T}
\end{aligned}
$$

The solution of (14) provides the optimum cost allocation strategy for the considered decentralized detection system.

As a special case, the Gaussian scenario is investigated. Suppose that the probability distributions of the observations are independent when the hypothesis is given, and the distribution of the $i$ th observation is denoted by $\mathcal{N}\left(\mu_{i 0}, \sigma_{x_{i}}^{2}\right)$ and $\mathcal{N}\left(\mu_{i 1}, \sigma_{x_{i}}^{2}\right)$ under hypothesis $H_{0}$ and hypothesis $H_{1}$, respectively. In addition, the distribution of the $i$ th measurement noise is given by $\mathcal{N}\left(0, \sigma_{m_{i}}^{2}\right)$, and the observations are independent of the measurement noise. For the sensors, the Bayes rule is employed assuming UCA and equally likely priors [13]. In this setting, the probability distribution of $u_{i}$ (i.e., the decision of the $i$ th sensor) given the hypotheses can be specified as follows:

$$
p_{j}\left(u_{i}\right)= \begin{cases}Q\left(\frac{(-1)^{j}\left(\mu_{i 0}-\mu_{i 1}\right)}{2 \sqrt{\sigma_{x_{i}}^{2}+\sigma_{m_{i}}^{2}}}\right) & \text { if } u_{i}=0 \\ Q\left(\frac{(-1)^{j}\left(\mu_{i 1}-\mu_{i 0}\right)}{2 \sqrt{\sigma_{x_{i}}^{2}+\sigma_{m_{i}}^{2}}}\right) & \text { if } u_{i}=1\end{cases}
$$

for $j \in\{0,1\}$, where $p_{j}\left(u_{i}\right)$ represents the probability of $u_{i}$ under hypotheses $H_{j}$. Hence, the optimization problem can be expressed for the Gaussian case as follows: $\min _{\left.\sigma^{2}\right\}_{K}}$

$$
\frac{1}{2} \sum_{z=t}^{K} \sum_{c=1}^{\left(\begin{array}{c}
K \\
z
\end{array}\right)} \prod_{i=1}^{K} Q\left(\beta_{(z, c, i)} \frac{\mu_{i 1}-\mu_{i 0}}{2 \sqrt{\sigma_{x_{i}}^{2}+\sigma_{m_{i}}^{2}}}\right)
$$

$\left\{\sigma_{m_{i}}^{2}\right\}_{i=1}^{K}$

$$
+\frac{1}{2} \sum_{z=0}^{t-1} \sum_{c=1}^{\left(\begin{array}{c}
K \\
z
\end{array}\right)} \prod_{i=1}^{K} Q\left(-\beta_{(z, c, i)} \frac{\mu_{i 1}-\mu_{i 0}}{2 \sqrt{\sigma_{x_{i}}^{2}+\sigma_{m_{i}}^{2}}}\right)
$$

subject to $\frac{1}{2} \sum_{i=1}^{K} \log _{2}\left(1+\frac{\sigma_{x_{i}}^{2}}{\sigma_{m_{i}}^{2}}\right) \leq C_{T}$

where $\beta_{(z, c, i)}=2 l_{(z, c, i)}-1$. The solution of this optimization problem leads to the optimal performance for the considered decentralized detection system by optimally allocating the cost values to the measurement devices (sensors).

In the following lemma, the convexity of the optimization problem in (16) is investigated for the special case of two sensors. (The proof is not presented due to the space limitation.)

Lemma 1: Consider the Gaussian scenario that leads to the optimization problem in (16). In addition, suppose that $K=2$, $\mu_{i 0}=0$, and $\mu_{i 1}=\mu>0$ for $i=1,2$. Then, the problem in (16) is a convex optimization problem if $\sigma_{x_{i}}^{2}+\sigma_{m_{i}}^{2} \leq \mu^{2} / 12$ for $i=1,2$ and for all values of $\sigma_{m_{i}}^{2}$ under the total cost constraint.

\section{NumERICAL RESUlts AND CONCLUSIONS}

In this section, the performance of the proposed optimal cost allocation strategy is evaluated via numerical examples. Firstly, the results for centralized detection are presented. The distribution of the observation $\mathbf{x}$ under hypothesis $H_{0}$ is given by $\mathcal{N}(\mathbf{0}, \boldsymbol{\Sigma})$, where $\mathbf{0}=[0,0,0]^{T}$. Similarly, the distribution of $\mathbf{x}$ under hypothesis $H_{1}$ is modeled as $\mathcal{N}(\mathbf{1}, \boldsymbol{\Sigma})$, where $\mathbf{1}=[1,1,1]^{T}$. In these distributions, $\boldsymbol{\Sigma}$ represents the covariance matrix, which is expressed as $\operatorname{diag}\left\{\sigma_{x_{1}}^{2}, \sigma_{x_{2}}^{2}, \sigma_{x_{3}}^{2}\right\}$. The values of the variances $\sigma_{x_{1}}^{2}, \sigma_{x_{2}}^{2}$ and $\sigma_{x_{3}}^{2}$ are set to 0.2 , 0.7 , and 1.2 , respectively. Measurement noise $\mathbf{m}$ also has Gaussian distribution denoted by $\mathcal{N}\left(\mathbf{0}, \boldsymbol{\Sigma}_{\boldsymbol{m}}\right)$, where $\boldsymbol{\Sigma}_{\boldsymbol{m}}=$ $\operatorname{diag}\left\{\sigma_{m_{1}}^{2}, \sigma_{m_{2}}^{2}, \sigma_{m_{3}}^{2}\right\}$.

The strategies that are compared with the proposed optimal cost allocation strategy are $(i)$ assignment of equal measurement variances to the measurement devices (sensors) and (ii) assignment of all the cost to the sensor with the best observation. When the measurement devices have equal measurement noise variances; i.e., $\sigma_{m}^{2}=\sigma_{m_{1}}^{2}=\sigma_{m_{2}}^{2}=$ $\sigma_{m_{3}}^{2}$, the variance $\sigma_{m}^{2}$ can be calculated by using the formula $\prod_{i=1}^{3}\left(1+\sigma_{x_{i}}^{2} / \sigma_{m}^{2}\right)=2^{2 C_{T}}$, where the variance $\sigma_{m}^{2}$ corresponds to the smallest positive root of this equation. After finding $\sigma_{m}^{2}$, the probability of error is calculated as

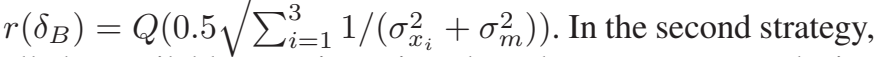
all the available cost is assigned to the measurement device having the observation with the smallest variance. In this example, $\sigma_{x_{1}}^{2}$ has the smallest variance, hence, all the cost is assigned to sensor 1 and $\sigma_{m_{1}}^{2}=\sigma_{x_{1}}^{2} /\left(2^{2 C_{T}}-1\right)$. The other variances $\sigma_{m_{2}}^{2}$ and $\sigma_{m_{3}}^{2}$ are set to infinity, and no measurements are taken from the corresponding measurement devices. The probability of error is obtained for this case as $r\left(\delta_{B}\right)=Q\left(0.5 \sqrt{2^{2 C_{T}}-1} / \sqrt{2^{2 C_{T} \sigma_{x_{1}}^{2}}}\right)$. The results obtained for centralized detection are presented in Fig. 3, which illustrates the probability of error versus the total 


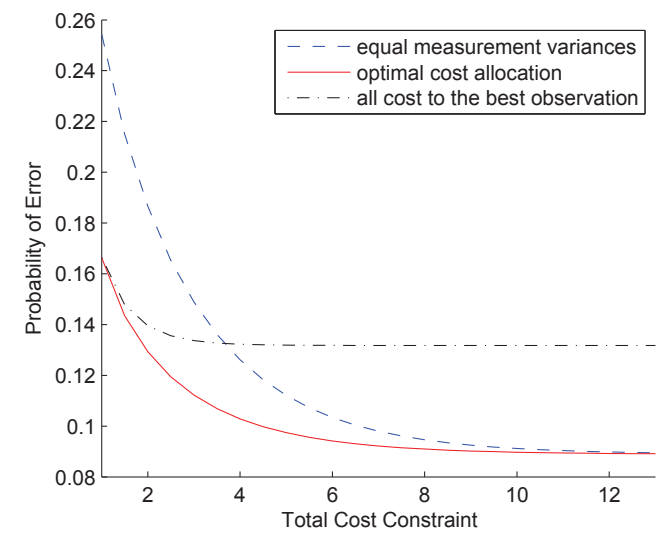

Fig. 3. Probability of error vs. total cost constraint for centralized detection.

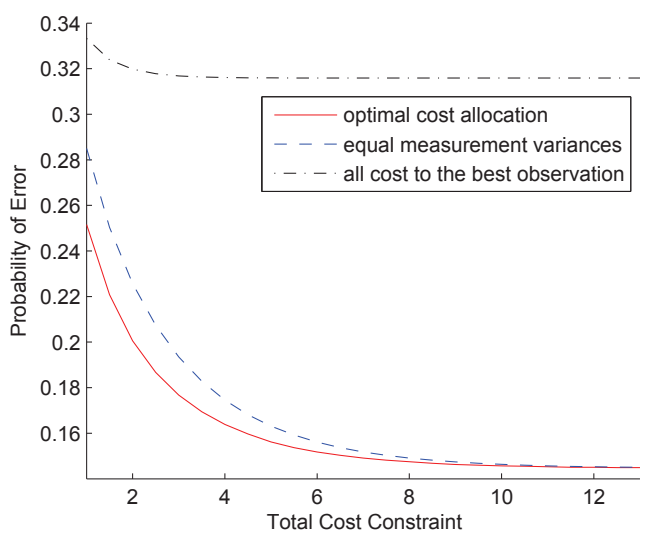

Fig. 4. Probability of error vs. total cost constraint for decentralized detection.

cost constraint, $C_{T}$, for the optimal cost allocation strategy and the two strategies described above. For small values of $C_{T}$, assigning all the cost to the sensor with the best observation converges the optimal solution since, when $C_{T}$ is small, the optimal strategy allocates the total cost to the sensors with the best observations. Moreover, the probability of error for assigning all the cost to the sensor with the best observation converges to $Q\left(0.5 / \sqrt{\sigma_{x_{1}}^{2}}\right)$, which is equal to $Q(0.5 / \sqrt{0.2})=0.1318$ since $\sigma_{m_{1}}^{2}$ goes to zero as $C_{T}$ increases. For high total cost constraints, the equal measurement variances strategy converges to the optimal strategy. Similar to the strategy that assigns all the cost to the sensor with the best observation, when $C_{T}$ is high, the measurement noise variances become low and the probability of error converges to $r\left(\delta_{B}\right)=Q\left(0.5 \sqrt{1 / \sigma_{x_{1}}^{2}+1 / \sigma_{x_{2}}^{2}+1 / \sigma_{x_{3}}^{2}}\right)$ which is equal to 0.0889 for the values specified above. Overall, the proposed optimal cost allocation strategy yields the lowest probabilities of error. In other words, the optimum performance is attained with the optimal cost allocation strategy.

For the same setting as in Fig. 3, the results for decentralized detection (Section III) are presented in Fig. 4. As observed from Fig. 4, assigning all the cost to the sensor with the best observation yields the worst performance in this case since all the sensors make their own decisions. When zero cost is assigned to a sensor, the measurement noise variance becomes infinity and the probability of error for that measurement device becomes 0.5 . Then, the probability of error converges to $r(\Gamma)=0.75 Q\left(0.5 / \sqrt{\sigma_{x_{1}}^{2}}\right)+0.5 Q\left(-0.5 / \sqrt{\sigma_{x_{1}}^{2}}\right)$ for high cost constraints. For $\sigma_{x_{1}}^{2}=0.2$, the probability of error converges to 0.3159 . When the cost constraint is high, the equal measurement variances strategy converges to the optimal strategy. For high cost constraints, the probability of error for the equal measurement variances strategy converges to $r(\Gamma)=a b+a c+b c-2 a b c$ where $a=Q\left(0.5 / \sqrt{\sigma_{x_{1}}^{2}}\right)$, $b=Q\left(0.5 / \sqrt{\sigma_{x_{2}}^{2}}\right)$, and $c=Q\left(0.5 / \sqrt{\sigma_{x_{3}}^{2}}\right)$. For the values specified above, $r(\Gamma)$ converges to 0.1446 . Overall, the optimal cost allocation strategy yields the lowest probabilities of error for decentralized detection, as well.

In this study, the optimal cost allocation problem has been investigated for Bayesian detection systems in the presence of cost constrained measurements. A cost allocation strategy has been proposed to minimize the probability of error for both centralized and decentralized detection systems. A closed form expression has been obtained for the measurement noise variances (equivalently, costs) in the case of centralized detection in the Gaussian scenario. For decentralized detection, a general probability of error term has been considered for the majority rule. In addition, the Gaussian scenario has been investigated as a special case, and the convexity property of the probability of error for the case of two local sensors has been stated.

\section{REFERENCES}

[1] C. Xu and S. Kay, "On centralized composite detection with distributed sensors," in IEEE Radar Conf., May 2008, pp. 1-6.

[2] J. N. Tsitsiklis, "Decentralized detection," in Advances in Signal Process. H. V. Poor and J. B. Thomas, Eds., vol. 2, pp. 297-344. JAI Press, 1993.

[3] A. Ozcelikkale, H. M. Ozaktas, and E. Arikan, "Signal recovery with cost-constrained measurements," IEEE Trans. Signal Process., vol. 58, no. 7, pp. 3607-3617, Jul. 2010

[4] B. Dulek and S. Gezici, "Cost minimization of measurement devices under estimation accuracy constraints in the presence of Gaussian noise," Digit. Signal Process., vol. 22, pp. 828-840, 2012.

[5] K. Liu and A.M. Sayeed, "Optimal distributed detection strategies for wireless sensor networks," in Proc. 42nd Annual Allerton Conf. Coтmиnications, Control Computing, Monticello, IL, Oct. 2004.

[6] J. F. Chamberland and V. V. Veeravali, "Decentralized detection in sensor networks," IEEE Trans Signal Process., vol. 51, pp. 407-416, Feb. 2003.

[7] W. P. Tay, "Decentralized Detection in Resource-Limited Sensor Network Architectures," Ph.D. dissertation, MIT, Cambridge, MA, 2007.

[8] J. F. Chamberland and V. Veeravalli, "Asymptotic results for decentralized detection in power-constrained wireless sensor networks," IEEE J. Sel. Areas Commun., vol. 22, no. 6, pp. 1007-1015, Aug. 2004.

[9] S. Appadwedula, V. V. Veeravalli, and D. L. Jones, "Energy-efficient detection in sensor networks," IEEE Journal on Selected Areas in Communications, vol. 23, no. 4, pp.693-702, Apr. 2005.

[10] B. Dulek and S. Gezici, "Average Fisher information maximisation in presence of cost-constrained measurements," Electronics Letters, vol. 47, no. 11, pp. 654-656, May 2011.

[11] C. Bruni, G. Koch, F. Papa, "Estimate accuracy versus measurement cost saving in continuous time linear filtering problems," Journal of the Franklin Institute, vol. 350, no. 5, pp. 1051-1074, June 2013.

[12] M. E. Tutay, S. Gezici, H. Soganci, and O. Arikan, "Optimal channel switching over Gaussian channels under average power and cost constraints," IEEE Trans. on Commun., vol. 63, pp. 1907-1922, May 2015.

[13] H. V. Poor, An Introduction to Signal Detection and Estimation. New York: Springer-Verlag, 1994.

[14] S. Boyd and L. Vandenberghe, Convex Optimization. Cambridge, U.K.: Cambridge Univ. Press, 2004.

[15] R. T. Rockafellar, Convex Analysis. Princeton University Press, 1970

[16] G. Ferrari and R. Pagliari, "Decentralized detection in sensor networks with noisy communication links," in F. Davoli, S. Palazzo, and S. Zappatore (Eds.), Distributed Cooperative Laboratories: Networking, Instrumentation, and Measurements, Springer, 2006, pp. 233-249.

[17] Q. Zhang, P. K. Varshney, and R. D. Wesel, "Optimal distributed binary hypothesis testing with independent identical sensors," in Conf. Information Sciences and Systems, Mar. 2000. 